\title{
MEJORAMIENTO DE LA PRODUCCIÓN DE LA PRADERA A TRAVÉS DEL MANEJO SILVOPASTORIL EN LOS BOSQUES DE ÑIRRE EN MAGALLANES.
}

\author{
Schmidt ${ }^{11}$, H., Olivares, A., Silva, C., Osses, T. y Schmidt, A.
}

\section{RESUMEN}

La superficie de bosques de ñirre (Nothofagus antarctica) en la región de Magallanes y la Antártica Chilena abarca aproximadamente 220.000 ha. En ellos la utilización ganadera no es eficiente. Como no se aplica un manejo silvopastoril las praderas se han deteriorado y hay dificultades para regenerar y conservar los bosques.

Para revertir el deterioro de los bosques y mejorar la productividad de la pradera, la Universidad de Chile inició una serie de ensayos para manejar los bosques con un criterio silvopastoril. Se analizó los efectos de la cobertura arbórea sobre las condiciones microclimáticas y la producción de la pradera. El ensayo consta de 4 tratamientos; un tratamiento testigo, un raleo, fajas y una situación extrema sin cobertura, en la que se eliminaron todos los árboles.

Los efectos microclimáticos más importantes que se obtuvo por la disminución de la cobertura fueron un incremento de la radiación disponible para la pradera y de la temperatura del aire. También aumentó la velocidad del viento 2,1 veces en el bosque raleado y 8 veces en el tratamiento sin cobertura arbórea. La producción del estrato herbáceo aumentó de $726 \mathrm{Kg} \mathrm{ha}^{-1}$ en el bosque testigo a $1.529 \mathrm{Kg} \mathrm{ha}^{-1}, 1.984 \mathrm{Kg} \mathrm{ha}^{-1}$ y $1951 \mathrm{Kg} \mathrm{ha}^{-1}$, uno, dos y cuatro años después del raleo, respectivamente. La proporción de especies forrajeras se elevó de $15 \%$ en el bosque testigo a $67 \%$ en el bosque raleado en el segundo año.

Palabras clave: Nothofagus antarctica, cobertura arbórea, producción de la pradera.

\section{SUMMARY}

The Nirre (Nothofagus antarctica) forests cover 220.000 ha in the region of Magallanes and Antartica Chilena. Current cattle practices are not efficient because forests are not silviculturally treated and cattle are managed in an extensive way. As result, the pastures have declined in quality and the conservation and the renovation of forests are more difficult than before. In order to revert the deterioration of forests and to improve the productivity of the prairies, the University of Chile started a series of scientific trials of managing forests with a silvopastoral criteria. The effects of tree canopy cover on microclimatic variables and pasture production were analyzed. A total of four treatments, consisting in a thinned stand, a clearcuted stand (100\% of trees cut), a strip-cutted stand, and a non-intervened stand (control), were selected.

The reduction in canopy cover increased incident solar radiation and air temperature. Wind speed increased 2.1 times in the thinned stand and 8 times in the clearcutted stand as compared with the control. Best production of forage species was found in the thinned stand. In this stand dry mass yield of grass species increased from 726 to 1529 and $1984 \mathrm{~kg} \mathrm{ha}^{-1}$ one, two and four years after thinning, respectively. The most important change was the gain in forage plant species which increased from $15 \%$ in the control stand to $67 \%$ in thinned stand.

Key words: Nothofagus antarctica, canopy cover, grass pasture, productivity.

\footnotetext{
${ }^{11}$ Facultad de Ciencias Forestales y de la Conservación de la Naturaleza, Universidad de Chile, Santa Rosa 11315, Santiago, Chile. hschmid@uchile.cl.
} 


\section{INTRODUCCIÓN}

El área de uso ganadero en la región de Magallanes y la Antártica Chilena comprende áreas de estepa con bajas precipitaciones y áreas cubiertas por matorrales, bosques y praderas naturales, donde las precipitaciones son mayores. En las áreas con precipitaciones entre 300 a 500 $\mathrm{mm}$ domina ñirre (Nothofagus antarctica) y en las zonas con mayor precipitación domina lenga (Nothofagus pumilio) y coihue de Magallanes (Nothofagus betuloides).

Los bosques de ñirre cubren una superficie de 220.000 hectáreas y se desarrollan en la zona de contacto con la estepa. En ellos la actividad principal es el uso ganadero, que no es eficiente, ya que no se manejan los bosques y las praderas. En consecuencia las praderas se han deteriorado y se observan dificultades en la renovación y la conservación de los bosques.

Para revertir el deterioro en los bosques de ñirre y mejorar la productividad de la pradera, la Universidad de Chile inició una serie de ensayos para manejar los bosques con un criterio silvopastoril. En ellos se analizó los efectos de la cobertura arbórea, las condiciones microclimáticas y la producción de la pradera. Se probaron cuatro tratamientos en una gradiente que va desde el bosque sin intervención, con cobertura alta, un raleo, fajas y una situación extrema sin cobertura, en la que se eliminó todos los árboles.

\section{MATERIAL Y MÉTODO}

\section{Área de estudio}

El ensayo se instaló en el año 2006, en la península Antonio Varas, en la provincia de Última Esperanza, frente a Puerto Natales, en un sector de bosques de ñirre con árboles dominantes de $14 \mathrm{~m}$ de altura que corresponde a un bosque secundario de aproximadamente 100 años de edad establecido después de un incendio. Este bosque antes de la intervención tenía una densidad de 1.065 árboles, un área basal de $26,7 \mathrm{~m}^{2}$ y un volumen de $155 \mathrm{~m}^{3}$ por hectárea.

\section{Tratamientos}

El ensayo consta de cuatro parcelas de una hectárea $(100 \mathrm{~m} \times 100 \mathrm{~m})$ con distintos tratamientos en la cobertura arbórea (Cuadro $\mathrm{N}^{\circ} 1$ y Figura $\mathrm{N}^{\circ} 1$ ). En cada tratamiento se instaló estaciones meteorológicas para medir la precipitación, la humedad relativa, la radiación solar, la temperatura del aire y la velocidad del viento. Los sensores fueron instalados a 1,7 $\mathrm{m}$ sobre el nivel del suelo, simulando la altura de los vacunos.

\section{Cuadro $\mathrm{N}^{\circ} 1$}

TRATAMIENTOS E INTERVENCIONES SILVÍCOLAS EN EL ENSAYO

\begin{tabular}{|l|c|}
\hline TRATAMIENTOS & INTERVENCIÓN SILVíCOLA \\
\hline 1. Testigo & Sin intervención silvícola \\
\hline 2. Raleo & Corta $60 \%$ del área basal \\
\hline $\begin{array}{c}\text { 3. Fajas alternas de } 5 \text { a } 20 \mathrm{~m} \text { de ancho } \\
-\quad \text { En una faja se cortó el bosque a tala rasa }\end{array}$ & $\begin{array}{c}\text { Corta } 100 \% \text { del área basal } \\
\text { Sin intervención silvícola }\end{array}$ \\
\hline$\quad$ En la otra faja se dejó el bosque sin intervenir & Corta $100 \%$ del área basal \\
\hline
\end{tabular}



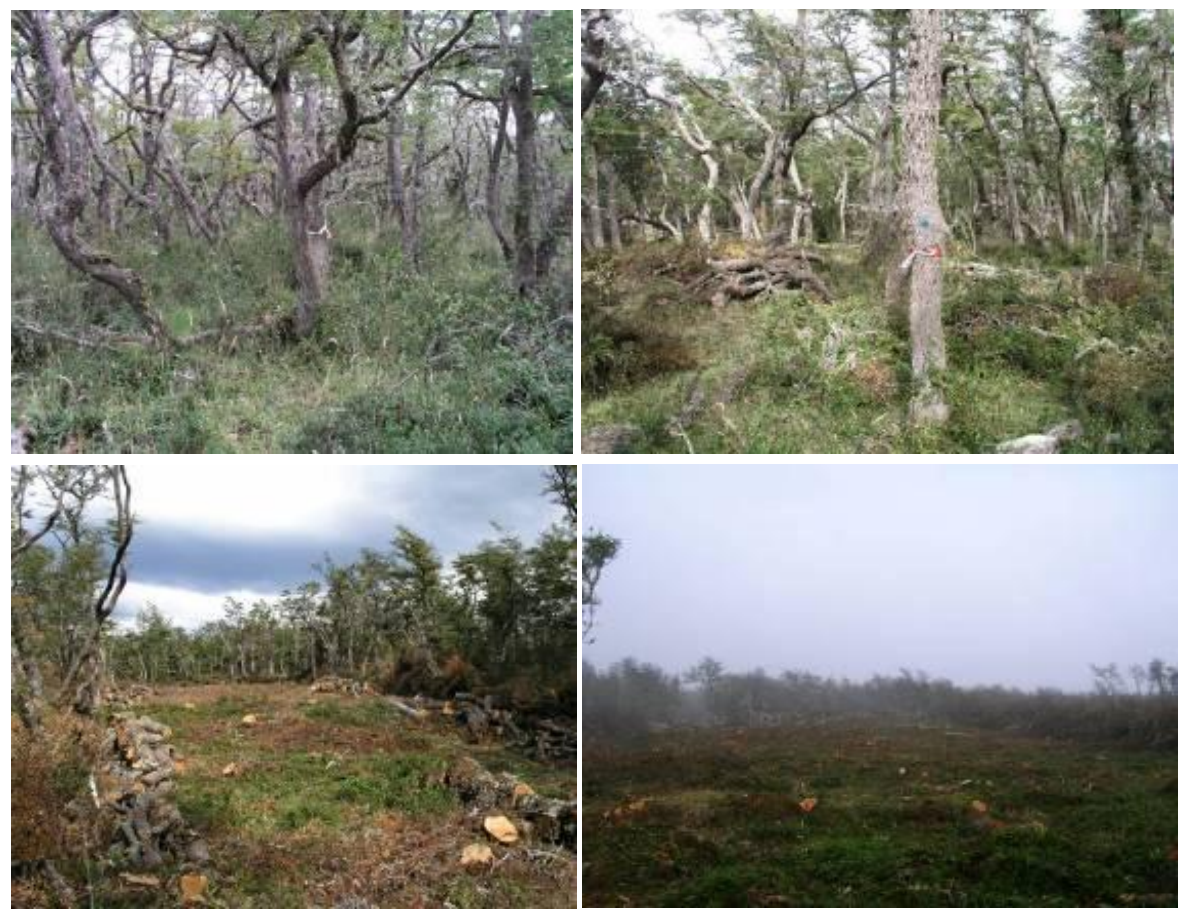

Testigo (arriba izq), Raleo (arriba der) Fajas (abajo izq) Tala Rasa (abajo der)

Figura $\mathrm{N}^{\circ} 1$

TRATAMIENTOS EN LA COBERTURA ARBÓREA EN EL ENSAYO ESTABLECIDO EN LA PENÍNSULA ANTONIO VARAS

\section{Inventario del Dosel Arbóreo en los Tratamientos}

Para medir la cobertura y las existencias en el bosque se distribuyó cuatro parcelas de 10 x 50 m en cada tratamiento (Figura $N^{\circ}$ 2). En las parcelas se midió el DAP, la altura de los árboles y la retoñación de los tocones de los árboles que fueron cortados. Para medir la cobertura del dosel arbóreo se tomó 20 fotografías hemisféricas en cada tratamiento, las que se analizaron con el software Hemi View. Para medir el efecto de la cobertura de los arbustos, en el tratamiento testigo se tomó 3 fotografías hemisféricas a distintas alturas por cada punto (a 1,0 m, 1,7 m y 2,3 m sobre el suelo).

De esta forma se obtuvo valores para el cielo visible, la radiación indirecta, directa y global, la cobertura de copas y el índice de área foliar.

En los tratamientos testigo, raleo y fajas alternas se instaló tres anemómetros, tres sensores de temperatura y tres sensores de humedad relativa conectados a módulos de regulación y almacenamiento de datos. La captura de los registros se hizo cada una hora. 


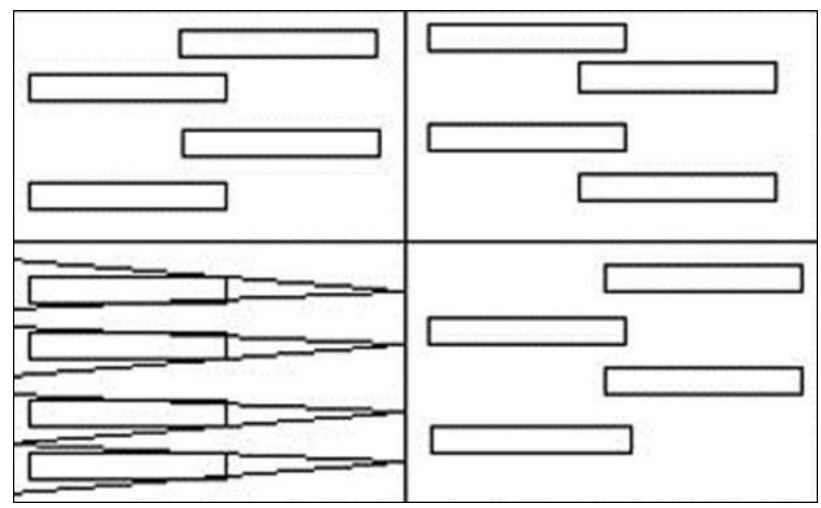

Testigo (arriba izq), Raleo (arriba der) Fajas (abajo izq) Tala Rasa (abajo der)

Figura $\mathrm{N}^{\circ} 2$

\section{ESQUEMA DISEÑO Y DISTRIBUCIÓN DE PARCELAS DE INVENTARIO EN} ENSAYO ESTABLECIDO EN LA PENÍNSULA ANTONIO VARAS

\section{Medición del Estrato Herbáceo}

Para medir el desarrollo del estrato herbáceo se instaló cuatro transectos de $100 \mathrm{~m}$ en cada tratamiento. En ellos se tomó 100 fotografías digitales, distanciadas a 4 m entre si.

Las fotografías fueron procesadas mediante una grilla de 16 puntos, en los que se midió la composición y la cobertura de las especies dominantes de la pradera, empleando un método similar al point cuadrat (Figura $\mathrm{N}^{\circ} 3$ ).

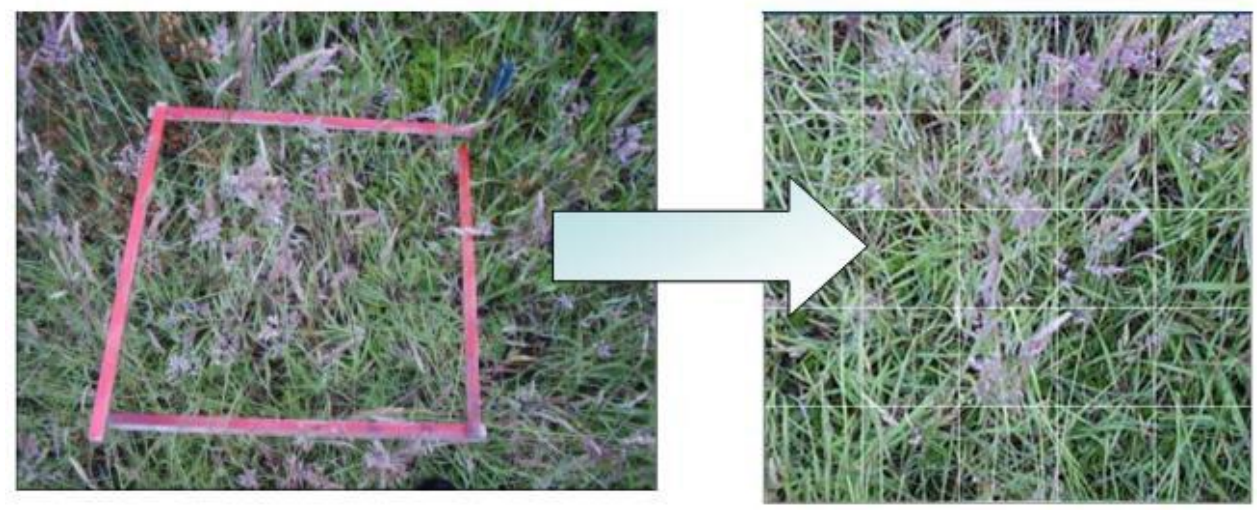

Figura $\mathbf{N}^{\circ} 3$

PROCESAMIENTO DE LAS SUB-PARCELAS DE COBERTURA DEL ESTRATO HERBÁCEO.

Para medir la producción de materia seca después de la intervención, se cosechó la pradera en 20 parcelas de un $\mathrm{m}^{2}$ en cada tratamiento. Los pastos fueron cosechados al final del período de crecimiento, en el año 2007, 2008 y 2010. 


\section{RESULTADOS Y DISCUSIÓN}

\section{Características del Bosque y los Arbustos Originales}

En el bosque se pudio determinar tres estratos. Un dosel superior conformado por las copas de los árboles a una altura dominante de $14 \mathrm{~m}$, un dosel de arbustos intermedio con alturas hasta 3 metros y un estrato de arbustos bajos y plantas herbáceas que cubre el piso (Figura $\mathrm{N}^{\circ} 4$ ). Las especies arbustivas encontradas fueron Escallonia serrata, Berberis buxifolia, Pernettya mucronata y Ribes magallanicum, las que tienen una altura promedio de $80 \mathrm{~cm}$
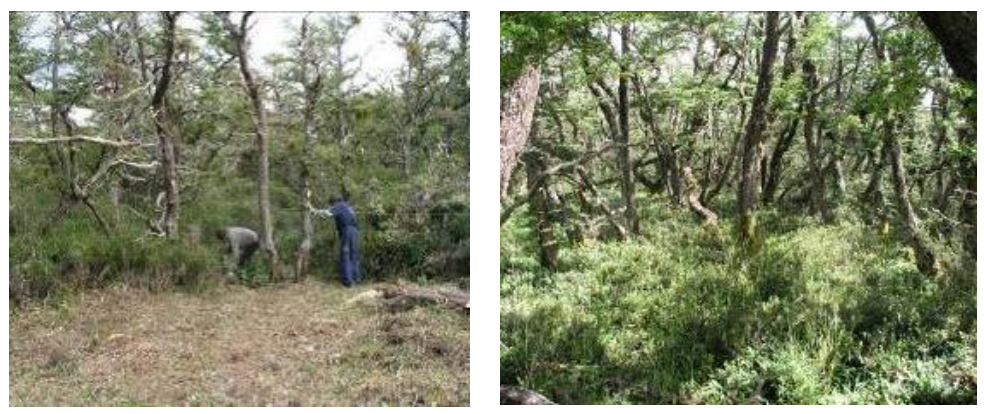

Figura $N^{\circ} 4$

ESTRUCTURA DEL BOSQUE DE ÑIRRE CON UN DOSEL SUPERIOR DE ÁRBOLES, UN ESTRATO ARBUSTIVO INTERMEDIO Y UN ESTRATO ARBUSTIVO Y HERBÁCEO EN EL PISO

\section{Distribución de la Radiación Disponible Bajo el Dosel Arbóreo y Arbustivo}

La radiación global disponible bajo el dosel arbóreo (medida a 2,3 m de altura) fue 44,6\% de la radiación disponible sobre el dosel. Bajo la cobertura del dosel arbustivo (medida a 1,0 m de altura) la radiación global disponible disminuye a $34,1 \%$ de la disponible sobre el dosel arbóreo. Esto implica una pérdida de casi $25 \%$ de la radiación disponible bajo el dosel arbóreo de los ñirres (Cuadro $\mathrm{N}^{\circ} 2$ ).

\section{Cuadro $\mathrm{N}^{\circ} 2$}

COBERTURA DE COPAS, ÍNDICE FOLIAR Y RADIACIÓN DISPONIBLE A DISTINTAS ALTURAS BAJO LOS ÁRBOLES Y LOS ARBUSTOS EN EL BOSQUE DE ÑIRRE TESTIGO EN EL ENSAYO.

\begin{tabular}{|l|c|c|c|c|c|c|}
\hline $\begin{array}{l}\text { Altura } \\
\text { Fotografía }\end{array}$ & $\begin{array}{c}\text { Cielo } \\
\text { Visible } \\
(\%)\end{array}$ & $\begin{array}{c}\text { Radiación } \\
\text { Indirecta } \\
(\%)\end{array}$ & $\begin{array}{c}\text { Radiación } \\
\text { Directa } \\
(\%)\end{array}$ & $\begin{array}{c}\text { Radiación } \\
\text { Global } \\
(\%)\end{array}$ & $\begin{array}{c}\text { Cobertura } \\
\text { de Copas } \\
(\%)\end{array}$ & $\begin{array}{c}\text { Índice } \\
\text { Área } \\
\text { Foliar } \\
\left(\mathbf{m}^{2} \mathbf{~ m}^{-2}\right)\end{array}$ \\
\hline $\mathbf{1 , 0} \mathbf{~ m}$ & $28,4^{\mathrm{a}}$ & $37,4^{\mathrm{a}}$ & $33,4^{\mathrm{a}}$ & $34,1^{\mathrm{a}}$ & $49,2^{\mathrm{a}}$ & $1,19^{\mathrm{a}}$ \\
\hline $\mathbf{1 , 7} \mathbf{~ m}$ & $31,0^{\mathrm{a}}$ & $40,1^{\mathrm{a}}$ & $36,5^{\mathrm{ab}}$ & $37,1^{\mathrm{ab}}$ & $43,3^{\mathrm{a}}$ & $1,13^{\mathrm{a}}$ \\
\hline $\mathbf{2 , 3} \mathbf{~ m}$ & $38,1^{\mathrm{b}}$ & $48,6^{\mathrm{b}}$ & $43,8^{\mathrm{b}}$ & $44,6^{\mathrm{b}}$ & $37,0^{\mathrm{a}}$ & $0,85^{\mathrm{b}}$ \\
\hline $\boldsymbol{F}$ & 13,86 & 9,99 & 4,17 & 5,24 & 2,11 & 7,84 \\
\hline $\boldsymbol{P}$ & 0,000 & 0,000 & 0,020 & 0,008 & 0,131 & 0,001 \\
\hline
\end{tabular}

F: Fisher test; $P$ : probabilty level; Letras indican diferencias significativas entre tratamientos $(p<0,05)$. 


\section{Coberturas y Existencias en el Bosque}

El bosque original del ensayo es un renoval constituido por 1.830 árboles por hectárea, con un área basal de $35,9 \mathrm{~m}^{2} \mathrm{ha}^{-1}$ y un volumen de $217 \mathrm{~m}^{3} \mathrm{ha}^{-1}$. En el raleo se extrajo un $70 \%$ de los árboles, correspondiente al $40 \%$ de las existencias. En el tratamiento de fajas alternas se cortó los árboles en el $50 \%$ de la superficie y en la tala rasa el $100 \%$. Un $40 \%$ de los tocones retoñó en los primeros meses después de la intervención (Cuadro $\mathrm{N}^{\circ} 3$ ).

Cuadro $\mathrm{N}^{\circ} 3$

COBERTURAS, EXISTENCIAS Y RETOÑOS DE LOS TOCONES DE ÑIRRE EN EL ENSAYO EN EL PRIMER AÑO DESPUÉS DE LA INTERVENCIÓN

\begin{tabular}{|l|c|c|c|c|c|c|}
\hline Tratamiento & $\begin{array}{c}\text { Densidad } \\
\left(\mathbf{a r b ~ h a}^{-1}\right)\end{array}$ & $\begin{array}{c}\text { Cobertura } \\
(\%)\end{array}$ & $\begin{array}{c}\mathbf{G} \\
\left(\mathbf{m}^{\mathbf{2}} \mathbf{h a}^{-1} \mathbf{)}\right.\end{array}$ & $\begin{array}{c}\mathbf{G} \text { extraído } \\
(\%)\end{array}$ & $\begin{array}{c}\text { Volumen } \\
\left(\mathbf{m}^{3} \mathbf{h a}^{-1}\right)\end{array}$ & $\begin{array}{c}\text { Retoños } \\
(\%)\end{array}$ \\
\hline Testigo & 1.830 & 57 & 35,9 & 0 & 217 & --- \\
\hline Raleo & 560 & 41 & 21,5 & 40 & 130 & 56,6 \\
\hline Fajas & $0-1.830$ & 30 & 18 & 50 & $0-217$ & 37,2 \\
\hline Tala rasa & 0 & 0 & 0 & 100 & 0 & 23,9 \\
\hline
\end{tabular}

\section{Mediciones Meteorológicas}

Los registros de los instrumentos en los años 2007 y 2008 son resumidos en el Cuadro $N^{\circ} 4$ (no se incluyen los registros de la tala rasa).

\section{Cuadro $\mathrm{N}^{\circ} 4$}

RESUMEN DE LOS REGISTROS DE LAS MEDICIONES METEOROLÓGICAS EN LOS AÑOS 2007 Y 2008 EN EL ENSAYO SILVOPASTORIL EN ANTONIO VARAS

\begin{tabular}{|c|c|c|c|c|c|c|c|}
\hline \multirow{2}{*}{ Variables } & \multirow{2}{*}{ Tratamiento } & \multicolumn{4}{|c|}{2007} & \multicolumn{2}{|c|}{2008} \\
\hline & & Verano & Otoño & Invierno & Primavera & Verano & Otoño \\
\hline \multirow{4}{*}{$\begin{array}{l}\text { Viento } \\
\left(\mathrm{m} \mathrm{s}^{-1}\right)\end{array}$} & Testigo & $0,31^{a}$ & $0,27^{a}$ & $0,20^{a}$ & $0,37^{\mathrm{a}}$ & $0,28^{a}$ & $0,21^{a}$ \\
\hline & & & & $-0,04$ & $-0,05$ & $-0,04$ & $-0,05$ \\
\hline & Fajas & $\begin{array}{l}0,45^{a} \\
-0,11\end{array}$ & $\begin{array}{l}0,34^{a} \\
(0,07) \\
\end{array}$ & $\begin{array}{l}0,33^{a} \\
-0,05\end{array}$ & $\begin{array}{l}0,56^{a} \\
-0,08\end{array}$ & $\begin{array}{c}0,45^{a b} \\
-0,09 \\
\end{array}$ & $\begin{array}{l}0,25^{a} \\
-0,08\end{array}$ \\
\hline & Raleo & $\begin{array}{l}0,72^{a} \\
-0,19 \\
\end{array}$ & $\begin{array}{l}0,82^{b} \\
(0,04) \\
\end{array}$ & $\begin{array}{l}0,61^{b} \\
-0,02 \\
\end{array}$ & $\begin{array}{l}0,96^{b} \\
-0,05 \\
\end{array}$ & $\begin{array}{l}0,78^{b} \\
-0,08 \\
\end{array}$ & -- \\
\hline \multirow[t]{3}{*}{$\begin{array}{c}\text { Temperatura } \\
\left({ }^{\circ} \mathbf{C}\right)\end{array}$} & Testigo & $\begin{array}{l}10,64^{\mathrm{a}} \\
(0,47) \\
\end{array}$ & $\begin{array}{l}3,45^{a} \\
(0,65) \\
\end{array}$ & $\begin{array}{l}2,86^{a} \\
-0,68 \\
\end{array}$ & $\begin{array}{l}8,26^{a} \\
-0,69 \\
\end{array}$ & $\begin{array}{c}12,04^{a} \\
(0,23) \\
\end{array}$ & $\begin{array}{l}2,78^{a} \\
-0,84 \\
\end{array}$ \\
\hline & Fajas & $\begin{array}{l}10,97^{a} \\
(0,51)\end{array}$ & $\begin{array}{l}3,76^{a} \\
(0,65)\end{array}$ & $\begin{array}{l}3,06^{a} \\
-0,63\end{array}$ & $\begin{array}{l}8,23^{a} \\
-0,75 \\
\end{array}$ & $\begin{array}{l}12,16^{a} \\
(0,23)\end{array}$ & $\begin{array}{l}3,02^{a} \\
-0,84 \\
\end{array}$ \\
\hline & Raleo & $\begin{array}{l}11,03^{a} \\
(0,48) \\
\end{array}$ & $\begin{array}{l}3,96^{a} \\
(0,64) \\
\end{array}$ & $\begin{array}{l}2,49^{a} \\
(0,94) \\
\end{array}$ & $\begin{array}{l}9,24^{a} \\
-0,72 \\
\end{array}$ & $\begin{array}{l}12,13^{a} \\
(0,26) \\
\end{array}$ & $\begin{array}{l}3,28^{a} \\
-0,82 \\
\end{array}$ \\
\hline \multirow{3}{*}{$\begin{array}{c}\text { Humedad } \\
\text { relativa } \\
(\%)\end{array}$} & Testigo & $\begin{array}{l}80,20^{a} \\
(1,88)\end{array}$ & $\begin{array}{l}90,45^{a} \\
(1,29)\end{array}$ & $\begin{array}{l}85,60^{a} \\
(2,25)\end{array}$ & $\begin{array}{c}75,76^{a} \\
-1,04 \\
\end{array}$ & $\begin{array}{l}73,44^{a} \\
(1,11)\end{array}$ & $\begin{array}{l}91,01^{\mathrm{a}} \\
(1,68)\end{array}$ \\
\hline & Fajas & $\begin{array}{c}74,75^{\text {ba }} \\
(1,25)\end{array}$ & $\begin{array}{l}86,97^{a} \\
(1,13)\end{array}$ & $\begin{array}{c}83,20^{a} \\
(1,85) \\
\end{array}$ & $\begin{array}{c}72,45^{a} \\
-1,84 \\
\end{array}$ & $\begin{array}{l}69,43^{a} \\
(1,56) \\
\end{array}$ & $\begin{array}{l}87,27^{a} \\
(2,05) \\
\end{array}$ \\
\hline & Raleo & $\begin{array}{l}70,80^{b} \\
(3,46)\end{array}$ & $\begin{array}{c}86,82^{a} \\
(1,18)\end{array}$ & $\begin{array}{l}84,90^{a} \\
(2,71)\end{array}$ & $\begin{array}{c}71,94^{a} \\
-1,07\end{array}$ & $\begin{array}{l}71,04^{a} \\
(1,06)\end{array}$ & $\begin{array}{l}88,42^{a} \\
(1,83)\end{array}$ \\
\hline
\end{tabular}

*Letras indican diferencias significativas entre tratamientos $(p<0,05)$. Los valores entre paréntesis corresponden al error estándar. 
En los tratamientos de disminución de la cobertura se observó un aumento en la velocidad del viento, al compararlas con la situación de bosque sin intervención (Cuadro $\mathrm{N}^{\circ} 4$ ). En el raleo el incremento fue de $210 \%$ y en las fajas aumentó $43 \%$.

En la tala rasa la velocidad del viento solo fue registrada durante los meses de marzo a mayo del año 2007. En ese período la intensidad del viento fue ocho veces mayor que en el bosque testigo.

La temperatura promedio en el bosque testigo fluctúa entre $12,1{ }^{\circ} \mathrm{C}$ en verano y $2,9{ }^{\circ} \mathrm{C}$ en invierno. Con la disminución de cobertura en los tratamientos no se generan diferencias significativas, solo se produce un leve aumento, que incide favorablemente en la productividad de la pradera y en los animales.

El incremento en el raleo fue de $0,4{ }^{\circ} \mathrm{C}, 6 \%$ más que en el bosque testigo, y en las fajas en promedio aumentó $0,2 \stackrel{\circ}{\circ} \mathrm{C}$. Con la disminución de cobertura en los tratamientos la humedad relativa baja levemente; en el bosque testigo es $83 \%$, baja a $79 \%$ en las fajas y en el raleo a $78 \%$ (Cuadro $\mathrm{N}^{\circ} 4$ ).

\section{Producción de Materia Seca en el Estrato Herbáceo}

El efecto de la disminución de la cobertura arbórea sobre la producción de materia seca en la pradera es presentado en el Cuadro $\mathrm{N}^{\circ} 5$.

La mayor producción se encontró en el bosque raleado, en el que se duplicó la producción de materia seca en el primer año después de la intervención en comparación con el bosque testigo. Esta diferencia se acentuó en el segundo año.

En promedio el rendimiento de materia seca en los tratamientos de raleo, fajas y tala rasa se incrementó un $18 \%$ respecto al primer año.

\section{Cuadro $\mathrm{N}^{\circ} 5$ \\ MATERIA SECA COSECHADA EN EL PRIMER Y SEGUNDO AÑO DESPUÉS DE LA INSTALACIÓN DEL ENSAYO}

\begin{tabular}{|c|c|c|c|}
\hline \multirow{3}{*}{ Tratamiento } & \multicolumn{3}{|c|}{ Materia Seca } \\
\hline & 2007 & 2008 & 2010 \\
\hline & \multicolumn{3}{|c|}{$\left(\mathrm{Kg} \mathrm{ha}^{-1}\right)$} \\
\hline Testigo & $726 \quad(60,3)$ & $557(23,3)$ & 1.054 \\
\hline Raleo & $1.529(98,6)$ & $1.984(57,4)$ & 1.951 \\
\hline Fajas & $1.192(110,8)$ & $1.124(32,8)$ & 1.428 \\
\hline Tala & $960 \quad(98,7)$ & $1.360(39,9)$ & 2.582 \\
\hline
\end{tabular}

Los valores entre paréntesis corresponden a la desviación estándar.

Al disminuir la cobertura arbórea se incrementó la proporción y el peso de las especies forrajeras, como Holcus lanatus (pasto miel) y Dactvlis glomerata (pasto ovillo) y tienden a disminuir especies poco interesantes como Blechnum penna-marina (helecho).

El peso de la materia seca de las forrajeras en el raleo, dos años después de la intervención, aumentó 16 veces (de 82 kilos por hectárea a 1.332 kilos). Algo similar ocurre en las fajas y en la tala rasa. 


\section{Cuadro $\mathrm{N}^{\circ} 6$}

\section{PRODUCCIÓN DE ESPECIES HERBÁCEAS EN LOS DISTINTOS TRATAMIENTOS DESPUÉS DE LA INTERVENCIÓN Y SU INTERÉS FORRAJERO}

\begin{tabular}{|c|c|c|c|c|c|c|}
\hline \multirow{2}{*}{ Tratamientos } & Holcus & Dactilis & Acaena* & Blechnum* & Otras ${ }^{*}$ & Total \\
\hline & \multicolumn{6}{|c|}{$\left(\mathrm{Kg} \mathrm{ha}^{-1}\right)$} \\
\hline Testigo & $57(24)^{a}$ & $25(9)^{a}$ & $31(9)^{a}$ & $422(94)^{a}$ & $22(6)^{a}$ & $557(104)^{a}$ \\
\hline Raleo & $1331(286)^{b}$ & $2(1)^{b a}$ & $52(17)^{a}$ & $551(117)^{a}$ & $48(11)^{a}$ & $1984(257)^{b}$ \\
\hline Faja & $1227(292)^{b}$ & $153(64)^{\mathrm{ca}}$ & $197(85)^{\text {ba }}$ & $577(134)^{a}$ & $95(49)^{a}$ & $2249(293)$ \\
\hline Tala Rasa & $616(184)^{b}$ & $86(54)^{\mathrm{ca}}$ & $175(40)^{b}$ & $428(99)^{a}$ & $55(21)^{a}$ & $1360(178)^{b}$ \\
\hline
\end{tabular}

Letras indican diferencias significativas entre tratamientos $(p<0,05)$.

Los valores entre paréntesis corresponden al error estándar.

* Especies sin interés forrajero

\section{CONCLUSIONES}

Los mejores resultados en la producción de la pradera fueron obtenidos reduciendo la cobertura del bosque, mediante raleos o fajas. La producción del estrato herbáceo prácticamente se duplicó en el segundo año y se triplicó después del tercer año. Sin embargo, más importante, es el aumento la proporción de especies forrajeras en esta producción, que se eleva 16 veces respecto al testigo. 
